The nearest approach to the production of the stereoscopic effect from a single picture is by photographing the illustration with its plane inclined at an angle of the optic axis of the lens, first in one direction, and then again in the opposite direction in which manner two dissimilar pictures are produced, which can be viewed with an ordinary stereoscope, and the result is by no means unsatisfactory. The process, however, is inconvenient.

For a long time past the writer has been experimenting with stereoscopy, and amongst other objects one has been to secure a stereoscopic effect with a single picture. This he has now succeeded in attaining in a comparatively simple manner.

If a stereoscopic slide is examined it will be found that each picture is compressed, so to speak, on the inner side, which is equivalent to saying that the central line of any one picture is shifted from the centre inwards, thus expanding one half of the picture and compressing the other half.

It therefore appeared to the writer that, if by some means a single picture could be made to give two images, each one com pressed in a suitable manner and then viewed with the ordinary stereoscopic lenses, the solid effect should be produced, and such is the case.

The method is as follows :-A large deep cylindrical lens is taken and cut in two along the line where the lens is thinnest. Thus two wedges are produced, each having one side curved and the other side flat. If these two pieces are placed together, with the thick portions towards one another, and held at a short distance over any picture, the eyes will perceive an image of the illustration in each lens, and the pictures will be compressed in the manner already referred to.

All that is now necessary is to view these two images with ordinary stereoscopic lenses. One picture will result, as solid in appearance as if the ordinary stereo slide had been employed.

The apparatus will be found of considerable use for looking at small photographic portraits, landscapes, \&c., as well as engravings. The result is very pretty.

Naturally one class of picture must suffer under the operation; but as these are comparatively rare, there is an extended use for the instrument, which is called the Pseudoscope.

The pictures unsuitable are those which represent an object when placed very near the eyes, since the image produced by solid objects in each eye in such cases differ greatly; but this difference in the image is comparatively small after a distance of, say, 15 to 20 feet. David Salomons.

\section{MAGNETIC OBSERVATIONS IN THE HARZ MOUNTAINS.}

$\mathrm{D}^{\mathrm{R}}$

R. ESCHENHAGEN has made an interesting series of magnetic observations at forty-two stations in the Harz Mountains.

The uncertainty of a declination observation was about $I^{\prime}$, of the dip with two needles about $I^{\prime} \cdot 2$, and the probable error of the horizontal force was about $\pm 0 \cdot 000$ o C.G.S. units. As it was intended to compare the results with those of a survey of the same district in which the deviations of the plumb-line were determined, the stations were selected with reference to this fact, and also to the geological conformation of the country.

The magnetic disturbances and disturbing forces were determined by a method essentially similar to that employed in the survey of the United Kingdom. The results were not very definite in the northern part of the district examined, but a clearly marked "ridge-line," or locus of attraction on the north-pole of the magnet, was discovered in the south. In one part of this the vertical disturbing force nearly reached 0.00400 C.G.S. units. There are few stations in England and Wales where the disturbance is as large as this, though at Stratford-onAvon it is exceeded by 25 per cent. At some places purely local attractions of very much larger magnitude were detected. Thus, on the Leistklippe the dip was altered by $I^{\prime}$, and the vertical disturbing force was 0.04224 C.G.S., or one-tenth of the whole vertical force. No such remarkable effects were observed near the principal granitic masses, such as the Brocken.

The fact that the magnetic ridge-line lies to the south of these is explained by the hypothesis that the granitic mass, of which the Brocken is the upper end, extends underground in a direction inclined to the vertical, and running from the surface towards the south. This view is, in the opinion of Dr. Eschenhagen, consistent with the geological facts. As, however, the granite is but slightly magnetic, it is also necessary to suppose that the heavier, more magnetic granite, lies deep, and is only brought near to the surface at a few points such as those at which the greatest local disturbances occur.

The local deviations of the plumb-line were deduced from observations made by the Royal Prussian Geodetic Institute. To the north of the mountains the plumb-line is deflected to the south ; from the south an attraction towards the north is detected. The line of no deviation is to the south of the Brocken, and runs nearly parallel to, but about $10-12 \mathrm{~km}$. north of, the magnetic ridge-line.

The remarkable result is therefore attained that ( $I$ ) geological evidence, (2) the magnetic needle, and (3) the plumb-line all point to the conclusion that the heavier rocks lie some distance to the south of the chief visible masses of granite.

Dr. Eschenhagen is careful to point out that it is unlikely that a similar agreement would be attained in every place where similar experiments might be carried out, as the rocks of greatest density are not necessarily magnetic. None the less, his observations make it probable that the magnet, the pendulum and the plunsb-line may add much to our knowledge of the details of the constitution of the crust of the earth; and his survey of the Harz Mountains is one of the most striking attempts which have yet been made to combine the results of magnetic and geodetic surveys.

A. W. R.

\section{THE JOURNAL OF ANATOMY AND PHYSTOLOGY.}

NEARLY nine years ago, on the occasion of the coming of age of the above-named Journal, we directed attention (NATURE, vol. xxxvii. p. 44I) to its association, as a medium of publication, with the then newly established "Anatomical Society of Great Britain and Ireland." Since that time the connection between the two has been maintained, much to the advantage of the Journal, for not only have the "Proceedings" of the Society materially improved and increased in bulk and importance, but many of the leading papers which have appeared in the body of the Journal have reached it through the mediation of the Society, while in the latter's annual reports of its "Committee of Collective Investigation" there have been contributed results of great service alike to the scientific and surgical anatomist. Owing to the death of the founder of the Journal and main supporter during its earlier years, the beloved and universally respected Sir G. Humphry, a reconstitution of the staff of its "conductors" was recently decided upon, and with the issue for October last a new series was accordingly commenced, Profs. Sir W. Turner and J. G. McKendrick, so long associated with its success, announcing that for the future they would be assisted by Profs. D. J. Cunningham, A. Macalister, and G. D. Thane. There has just reached us the second (January) part which has appeared under these new auspices. During the years which have elapsed since our previous notice was written, three of the five present conductors of the Journal have been presidents of the Anatomical Society, while Prof. Macalister, having been on November 26 elected to that office, now combines the two functions. And during the same period the Anatomical Society undertook the preparation, and the conductors of the Journal the publication, of a couple of analytical indexes to its first thirty volumes, entrusting the task to Mr. A. W. Kappel, the efficient librarian of 